\title{
Hard X-ray monitoring of HMXB with Swift-BAT: a systematic search to reveal long term periodicities
}

\author{
G. Cusumano ${ }^{* a}$ V. La Parola, ${ }^{a}$ A. Segreto, ${ }^{a}$ P. Romano, ${ }^{a}$ S. Vercellone,${ }^{a}$ A. D'Aí, ${ }^{b}$ N. \\ Robba, ${ }^{b}$ \\ ${ }^{a}$ INAF, Istituto di Astrofisica Spaziale e Fisica Cosmica, Via U. La Malfa 153, I-90146 Palermo, \\ Italy \\ ${ }^{b}$ Dipartimento di Scienze Fisiche ed Astronomiche, Università di Palermo, via Archirafi 36, \\ 90123 Palermo, Italy \\ E-mail: cusumanodifc.inaf.it
}

The Burst Alert Telescope on board Swift has been performing a continuous monitoring of the sky in the hard X-ray energy range (15-150 keV) since November 2004. The telescope, thanks to its large field of view (1.4 sr half-coded) and its pointing strategy, covers a fraction between 50 and 80 per cent of the sky every day. This has allowed the quite continuous monitoring of many Galactic sources. The analysis of their long term light curves allows us to investigate the intrinsic emission variability, search for long periodicities (orbital periods) and discover the presence of eclipse events. The role of Swift-BAT is therefore fundamental to unveil the geometry of those binary systems (recently discovered by INTEGRAL) that, because of the high value of their absorption column density along the line of sight, are undetected by softer X-ray all-sky monitors. We present some timing results obtained on IGR sources through the BAT monitoring.

8th INTEGRAL Workshop "The Restless Gamma-ray Universe"

September 27-30 2010

Dublin Castle, Dublin, Ireland

\footnotetext{
${ }^{*}$ Speaker.
} 


\section{Introduction}

The IBIS/ISGRI telescope [1] on board the INTEGRAL satellite [2] has allowed the detection of a large number of new sources $(\sim 500)$ most of which characterized by a strong intrinsic absorption $\left(\mathrm{N}_{H}>10^{22} \mathrm{~cm}^{2}\right)$. About $\sim 26 \%$ of these source are associated with Galactic sources and in particulat 56 sources are identified as binary systems with a supergiant companion.

The Burst Alert Telescope (BAT, [3]) on board the Swift satellite [4], thanks to its large field of view (1.4 steradian half coded) and its pointing strategy covers a fraction of between $50 \%$ and $80 \%$ of the sky every day allowing a long and continuous monitoring in the X-ray energy range $15-150 \mathrm{keV}$. The BAT data offer an inportant tool to investigate the intrinsic emission variability of these sources, providing the history of their long-term emission with a good time coverage and in an energy range where absorption does not prevent their detection. We are performing a systematic study of the BAT light curves of the new INTEGRAL HMXB sources to search for orbital period periodicities. In this paper we present some timing results on the following INTEGRAL sources: IGR J16493-4348, IGR J16465-4507, IGR J05007-7047 and IGR J17354-3255.

\section{BAT survey data}

The results presented in this paper are obtained by the analysis of the BAT survey data collected during the first 54 months of the Swift mission. These data were processed with a dedicated software [5] that performs screening, mosaicking and source detection on data from coded mask instruments. IGR J16493-4348, IGR J16465-4507, IGR J05007-7047 and IGR J17354-3255 were detected in the 54 months BAT all-sky sky map with a significance maximized in the $15-50 \mathrm{keV}$ band of 20.8, 13.7, 16.1 and 18.9 standard deviations, respectively.

\section{Timing Analysis}

\subsection{Method}

Timing analysis was performed by applying the folding technique on the $15-50 \mathrm{keV}$ BAT light curves and searching in the period range between 0.5 and 100 days. The average rate in each profile phase bin was evaluated by weighting the rates by the inverse square of its statistical error

$$
R_{j}=\frac{\Sigma r_{i} / e r_{i}^{2}}{\Sigma 1 / e r_{i}^{2}}
$$

where $R_{j}$ is the average rate in the $\mathrm{j}$-th phase bin of the trial profile, $r_{i}$ is the rate of the light curve bin whose phase falls into the $\mathrm{j}$-th phase bin and $e r_{i}$ is the statistical error. The error on $R_{j}$ is $\left(\sqrt{\Sigma 1 / e r_{i}^{2}}\right)^{-1}$.

\subsection{IGR J16493-4348}

This source was discovered by INTEGRAL in 2004 [6]. A follow-up observation with Chan$d r a$ found a soft X-ray counterpart $\left(\mathrm{RA}(\mathrm{J} 2000)=16^{h} 49^{m} 26.92^{s} ; \operatorname{Dec}(\mathrm{J} 2000)=-43^{\circ} 49^{\prime} 8.96^{\prime \prime}\right.$ [7] $)$ allowing the optical association with 2MASS J1642695-4349090, a B0.5 Ib supergiant [8]. 


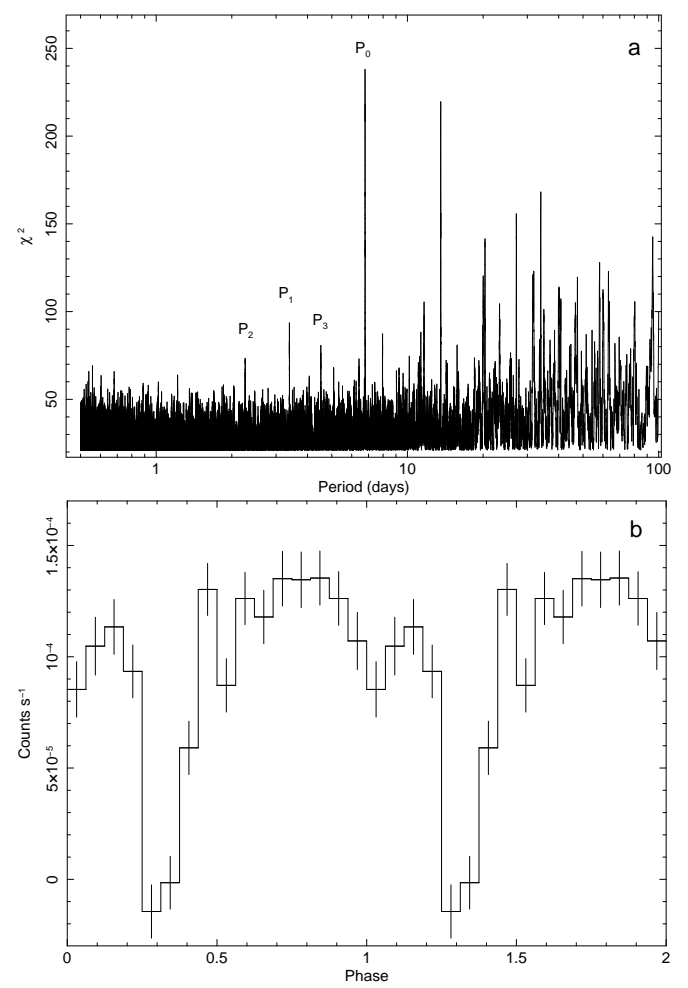

Figure 1: a: Periodogram of Swift-BAT (15-50 keV) data for IGR J16493-4348. b: Light curve folded at a period $\mathrm{P}_{0}=6.783$ days, with 16 phase bins.

The periodogram (Fig. 1, (a)) obtained by the folding procedure on the BAT light curve shows several features. The highest, with a $\chi^{2}$ value of $\sim 240$, is at $\mathrm{P}_{0}=6.782 \pm 0.002 \mathrm{~d}$. The error is the period resolution evaluated as $\mathrm{P}^{2} /\left(N \Delta T_{B A T}\right) \mathrm{d}$, where $N=16$ is the number of trial profile phase bins, and $\Delta T_{B A T}$ is the data span length ( $\sim 20.000$ days). The significance of $\mathrm{P}_{0}$ is evaluated as $\sim 8.5$ standard deviations in Gaussian statistics. We also see other evident features at periods multiple of $\mathrm{P}_{0}$ and at period tied to $\mathrm{P}_{0}$ as $\mathrm{P}_{1}\left(=\mathrm{P}_{0} / 2\right)$ due to the presence of the deep eclipse, still visible in the light curve folded at half of the period, $\mathrm{P}_{2}\left(\sim 2.27 \mathrm{~d}=\left(1 / \mathrm{P}_{0}+1 / \mathrm{P}_{1}\right)^{-1}\right)$ and $\mathrm{P}_{3}$ $\left(\sim 4.54 \mathrm{~d}=\left[\left(1 / \mathrm{P}_{0}+1 / \mathrm{P}_{1}\right) / 2\right]^{-1}\right)$ due to beat frequencies between $\mathrm{P}_{0}$ and $\mathrm{P}_{1}$. The pulsed profile (Fig. 1, b) folded at $\mathrm{P}_{0}$ with $\mathrm{T}_{\text {epoch }}=54173.757$ MJD shows a flat intensity level, abruptly broken by a deep full eclipse at phase $0.319 \pm 0.015$ corresponding to MJD (54175.92 \pm 0.10$) \pm n \times \mathrm{P}_{0}$ MJD.

\subsection{IGR J16465-4507}

IGR J16465-4507. This source was discovered by INTEGRAL in 2004 [9]. Follow-up observations with XMM-Newton revealed pulsations at $228 \pm 6 \mathrm{~s} \mathrm{[10]} \mathrm{and} \mathrm{allowed} \mathrm{the} \mathrm{identification} \mathrm{of}$ the optical counterpart with 2MASS J16463526-4507045 [11] classified as a B0.5 Ib supergiant at a distance of $\sim 8 \mathrm{kpc}$ [12] or as a O9.5 Ia supergiant at a distance of $9.5_{-5.7}^{+14.1} \mathrm{kpc}$ [13].

Figure 2 (a) shows the periodogram obtained folding the BAT data of IGRJ16465-4507. We find significant evidence for periodicity $\left(\chi^{2} \sim 270\right)$ at a period of $P_{0}=30.243 \pm 0.035$ days (the two features at higher periods are multiple of $P_{0}$ ). The significance of $P_{0}$ is 3.7 standard deviations. 


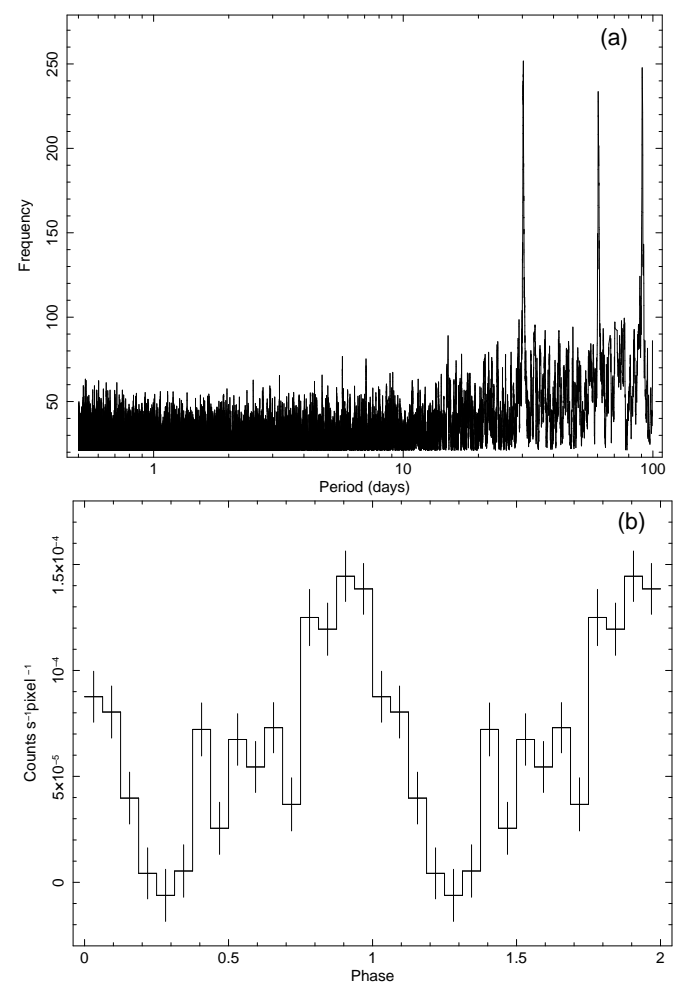

Figure 2: a: Periodogram of Swift-BAT (15-50 keV) data for IGR J16465-4507. b: Light curve folded at a period $P_{0}=30.243$ day, with 16 phase bins.

Figure 2 (b) shows the pulsed profile folded at $P_{0}$. There is clear evidence for a large phase interval ( $\sim 20 \%$ ) with an emission intensity consistent with zero, whose epoch centroid, evaluated by fitting the data around the dip with a Gaussian function, is at phase $0.297 \pm 0.015$, corresponding to MJD (52730.6 \pm 0.5$) \pm n P_{\text {orb }}$.

\subsection{IGR J05007-7047}

IGR J05007-7047, located in the Large Magellanic Cloud, was detected with INTEGRAL in the 17-60 keV band [14] with a flux of $1.2 \times 10^{-11} \mathrm{erg} \mathrm{cm}^{-2} \mathrm{~s}^{-1}$, corresponding to a luminosity of $3.6 \times 10^{36} \mathrm{erg} \mathrm{s}^{-1}$, assuming a distance of $50 \mathrm{kpc}$. A dedicated Chandra observation allowed its association with the relatively bright blue (V=14.8, B-V=-0.01) star USNO-B1.0 0192-0057570 [15] of spectral type B2 III [16] at a redshift consistent with that of LMC.

Figure 3 (a) shows the periodogram obtained for IGR J05007-7047. We find significant evidence for the presence of a periodicity $\left(\chi^{2} \sim 210\right)$ at a period of $P_{0}=30.77 \pm 0.03$ days. The other two significant features in the periodogram correspond to $2 \mathrm{P}_{0}$ and $3 \mathrm{P}_{0}$. The significance of $P_{0}$ is $\sim 7$ standard deviations. The pulsed profile (Fig. 3, b) folded at $\mathrm{P}_{0}$ with $\mathrm{T}_{\text {epoch }}=54159.75324287$ MJD, shows a roughly sinusoidal modulation with a minimum value consistent with zero intensity. The centroid of the minimum, evaluated by fitting the data around the dip with a Gaussian model is at phase $0.23 \pm 0.02$ corresponding to $\operatorname{MJD}(54166.8 \pm 0.6) \pm n \times \mathrm{P}_{0} \mathrm{MJD}$. 


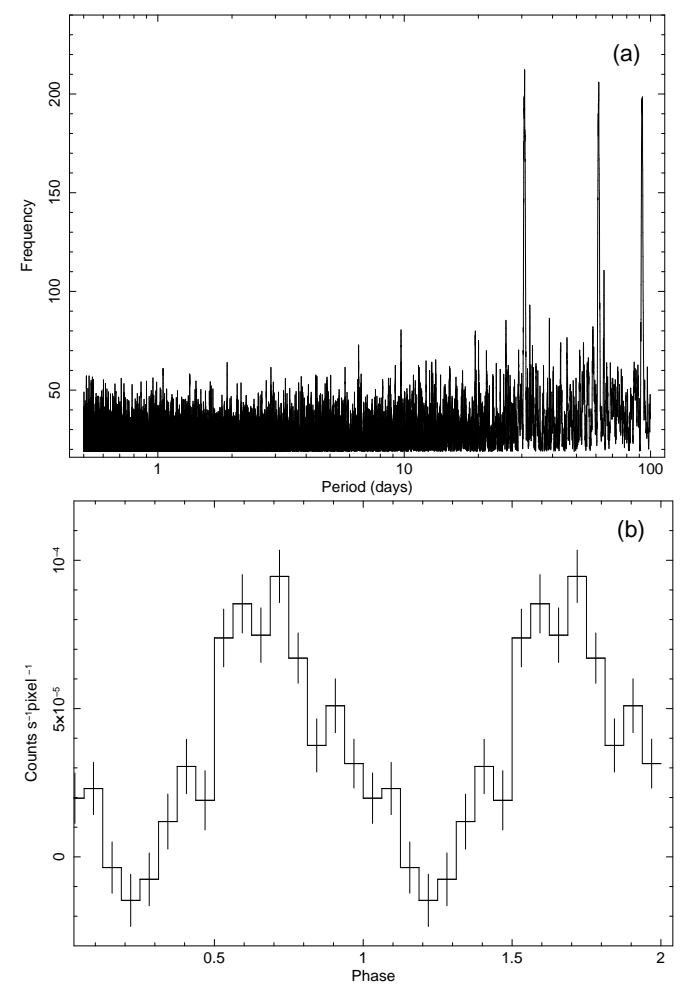

Figure 3: a: Periodogram of Swift-BAT (15-50 keV) data for IGR J05007-7047. b: Swift-BAT Light curve folded at a period $P=30.77 \pm 0.01$ day, with 16 phase bins.

\subsection{IGR J17354-3255}

IGR J17354-3255 was discovered during the INTEGRAL Galactic Bulge monitoring program $[17,18]$. The source has also a possible, highly variable, $\gamma$-ray counterpart detected with the Agile satellite (AGL J1734-3310, [19]).

The periodogram obtained for IGR J17354-3255 (Figure 4, a) shows significant evidence for the presence of a periodicity $\left(\chi^{2} \sim 121\right)$ at a period of $P_{0}=8.448 \pm 0.002$ days. The other significant features that appear in the periodogram corresponds to multiples of $\mathrm{P}_{0}$ (up to $8 \mathrm{P}_{0}$ ). The significance of $P_{0}$ is $\sim 4$ standard deviations. The pulsed profile (Fig. 4, b) folded at $\mathrm{P}_{0}$ with $\mathrm{T}_{\text {epoch }}=54175.15952181 \mathrm{MJD}$, shows a modulation with a minimum value consistent with zero intensity. The centroid of the minimum, evaluated by fitting the data around the dip with a Gaussian model is at phase $0.78 \pm 0.02$ corresponding to MJD $(54181.75 \pm 0.17) \pm n \times \mathrm{P}_{0} \mathrm{MJD}$.

\section{References}

[1] F. Lebrun, J.P. Leray, P. Lavocat, et al., A\&A 411, L141 (2003).

[2] C. Winkler, T.J.L. Courvoisier, G. Di Cocco, et al. A\&A 411, L1 (203)

[3] S. D. Barthelmy, L. M. Barbier, J. R. Cummings, et al., Space Science Reviews 1204, 143 (2005).

[4] N. Gehrels, G. Chincarini, P. Giommi, et al. ApJ, 611, 1005 (2004) 

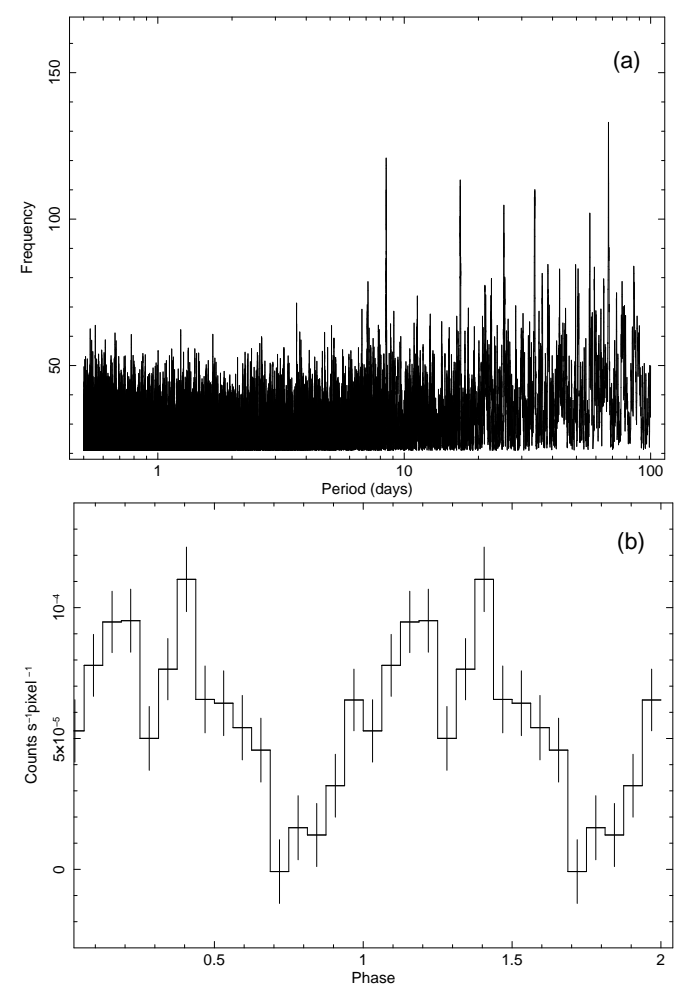

Figure 4: a: Periodogram of Swift-BAT (15-50 keV) data for IGR J17354-3255. b: Swift-BAT Light curve folded at a period $P=8.452 \pm 0.002$ day, with 16 phase bins.

[5] A. Segreto, G. Cusumano, C. Ferrigno, et al., $A \& A$ 510, 47 (2010)

[6] A. J. Bird, E.J. Barlow, L. Bassani, et al. ApJ 607, L33 (2004)

[7] L. Kuiper, P. Jonker, W. Hermsen, K. O’Brien ATel 654 (2005)

[8] E. Nespoli, J. Fabregat, R. E. Mennickent, ATel 1396 (2008)

[9] A. Lutovinov, J. Rodrigues, C. Budtz-Jorgensen, et al., ATel 329, 1 (2004)

[10] A. Lutovinov, M. Revnivtsev, M. Gilfanov, et al., A\&A 444, 821 (2005)

[11] J. A. Zurita Heras, R. Walter, ATel 336 (2004)

[12] I. Negueruela, D. M. Smith, J. M. Torrejon, P. Reig, ESA Special Publication 622, 255 (2007)

[13] E. Nespoli, J. Fabregat, R.E. Mennickent, $A \& A$ 486, 911 (2008)

[14] S. Sazonov, E. Churazov, M. Revnivtsev,A. Vikhlinin, R. Sunyaev, R. A\&A 444, L37 (2005)

[15] J.P. Halpern, ATel 572 (2005)

[16] N. Masetti, L. Morelli, E. Palazzi, et al., A\&A 459, 21 (2006)

[17] E. Kuulkers, S. Shaw, A. Paizis, A., et al., ATel 874 (2006)

[18] E. Kuulkers, S. Shaw, A. Paizis, et al., A\&A 466, 595 (2007)

[19] A. Bulgarelli, F. Gianotti, M. Trifoglio, et al., ATel 2017 (2009) 